\title{
Mechanism of the Protective Effect of Yulangsan Flavonoid on Myocardial Ischemia/Reperfusion Injury in Rats
}

\author{
Xudong Zhanga,b Xingmei Liang ${ }^{a, c} \quad$ Xing Lin ${ }^{a} \quad$ Shijun Zhang ${ }^{a}$ Zhongshi Huang ${ }^{a}$ \\ Chunxia Chen $^{\mathrm{a}}$ Youjia Guo ${ }^{\mathrm{a}}$ Feifei Xuan ${ }^{\mathrm{a}}$ Xiaohui Xua Renbin Huang ${ }^{\mathrm{a}}$ \\ aDepartment of Pharmacology, Guangxi Medical University, Nanning, 'Department of Physiology, \\ Mudanjiang Medical College, Mudanjiang, 'Department of Pharmacy, Guangxi Medical College, \\ Nanning, PR China
}

\section{Key Words}

$\mathrm{YLSF} \cdot \mathrm{MI} / \mathrm{RI} \cdot$ Lipid peroxidation $・ \mathrm{NOS} \cdot$ Apoptosis

\begin{abstract}
Aims: Effect and mechanism of Yulangsan flavonoid (YLSF) on rat myocardial ischemia/ reperfusion injury (MI/RI) has been investigated. Methods: Sprague-Dawley (SD) rats were randomly divided into seven groups (sham group, model group and NS group: $2 \mathrm{~mL}$ of normal saline/kg body weight was administered; diltiazem group: $5 \mathrm{mg}$ of diltiazem hydrochloride/kg body weight was administered; YLSFL, YLSFM and YLSFH groups: 20, 40 and $80 \mathrm{mg}$ of YLSF/ $\mathrm{kg}$ body weight was administered) and the MI/RI model was established. Myocardial infarct area, levels of myocardial enzymes and nitric oxide synthase (NOS) were measured. Caspase-3 and adenine nucleotide translocator-1 (ANT1) mRNA expression were evaluated by reverse transcription polymerase chain reaction (RT-PCR). Pathological structure and cardiocyte ultrastructure were also analysed. Results: Compared with the MI/RI group, pretreatment with YLSF or diltiazem hydrochloride decreased the infarct area, levels of inducible nitric oxide synthase (iNOS), caspase-3 as well as the leakage of myocardial enzyme and increased activities of total nitric oxide synthase (tNOS) as well as constitutive nitric oxide synthase (cNOS). Cellular edema and the infiltration of inflammatory cells were alleviated. Conclusions: The experiment showed that YLSF protected the heart against MI/RI, possibly by reducing lipid peroxidation damage, regulating NOS activity and modulating the apoptosis genes expression.
\end{abstract}

X. Zhang and X. Liang contributed equally to this work. 


\section{Introduction}

Myocardial ischemia/reperfusion injury (MI/RI) is the most common cause of cardiac morbidity and mortality in many countries, and research on this subject has become a focus in recent years [1]. MI/RI refers to the phenomenon by which reperfusion into the ischemic myocardial tissues aggravates the injury of myocardial structure and function [2], which can lead to arrhythmia, heart hypofunction, cardiocyte apoptosis, and other disorders [3]. The pathogenesis of MI/RI is apparently multifactorial, including the following steps: (1) intracellular acidosis, loss of intracellular $\mathrm{K}^{+}$, and accumulation of metabolites; (2) intra-cellular $\mathrm{Ca}^{2+}$ overload, loss of gap junction expression/function, and irreversible cellular injury; and (3) elevated levels of oxidative stress, progressive accumulation of reactive oxygen species (ROS), and mitochondrial dysfunction [3]. Furthermore, research has demonstrated that apoptosis plays an evident role in MI/RI and might be an important part of pathogenesis [4]. In addition, many studies have confirmed that the overproduction of free radicals, intracellular calcium overload, mitochondrial injury and inflammatory cell infiltration induced by MI/RI are related to the activation of apoptosis genes [5-6]. Therefore, research on the effect of YLSF on the expression of apoptosis genes such as caspase- 3 and ANT1 is consequential.

Over the years, major advances have been made toward understanding the role of nitric oxide (.NO) in the ischemic biology of the heart, and it has become clear that.NO represents one of the most important defenses against myocardial ischemia and reperfusion injury [78]. .NO can be generated by NOS, which catalyzes the conversion of L-arginine to .NO and L-citrulline [9]. NOS has an inducible NOS (iNOS) isoform and a constitutive NOS (cNOS) isoform. In addition, cNOS includes two isoforms: neuronal NOS (nNOS) and endothelial NOS (eNOS) [10].

According to current studies, .NO is an important regulator of myocardial function and vascular tone under physiological conditions. .NO is a simple molecule produced primarily by endothelial cells in the cardiovascular system and plays an important regulatory role in apoptotic cell death. .NO plays a vital role in the heart under physiological conditions via its involvement in maintaining coronary vasodilatory tone [11], inhibiting platelet aggregation [12] and the adhesion of neutrophils and platelets to vascular endothelium [13-15] and so on. The main source of cardiac.NO is generated through eNOS expressed by coronary endothelial cells and cardiac myocytes, whereas iNOS expression is low or absent in uninfected hearts [16, 17].YLSF is a specific and eutherapeutic product prepared from the roots of Millettia pulchra (Benth.) Kurz var. Laxior (Dunn) Z. Wei (Papilionaceae) that has been used in traditional folk medicine in Guangxi province for many years ${ }^{[18]}$. The roots are widely used to treat anemia, dizziness, hyposomnia, infantile malnutrition, stroke hemiplegia and so on [18, 19]. Previous studies have demonstrated that YLSF can protect the heart against myocardial ischemia in vitro and in vivo, is capable of scavenging hydroxyl radicals and oxyradicals, has antihypoxic and anti-stress properties, improves immunity and is also anti-hypertensive [20, 21]. However, whether the generation of NOS is modulated by YLSF and its down-regulation expression of apoptosis-related genes such as caspase-3 and ANT1 remains unconfirmed, which limits the full understanding of the effect of YLSF.

\section{Materials and Methods}

\section{Drugs and reagents}

YLSF was obtained from the Department of Pharmacology of Guangxi Medical University [22]. 500g decoction pieces of YLS (lot number: YLS20060302) was smashed to coarse powder and extracted with microwave-assisted extraction method for $20 \mathrm{~min}$ (extract solvent: 4L 60\% alcohol; extract temperature: $<60^{\circ} \mathrm{C}$; microwave power: $240 \mathrm{~W}$ ), then the method of concentration under reduced pressure and vacuum freeze-drying were used to prepare total YLSF. Concentrated ammonia reaction, aluminium chloride reaction, magnesium acetate reaction and hydrochloric acid magnesium powder reaction were used to identify the extract to be flavonoids. 
Evans blue and tetrazolium chloride (TTC) were obtained from Shanghai Chemical Reagent Co., Ltd. NOS, malondialdehyde (MDA), superoxide dismutase (SOD) and glutathione peroxidase (GSH-Px) detection kits were obtained from the Nanjing Jiancheng Bioengineering Institute. Aspartate aminotransferase (AST), creatine kinase (CK), creatine kinase-MB (CK-MB), lactate dehydrogenase (LDH) and lactate dehydrogenase1 (LDH1) were purchased from Randox Laboratories Ltd. (England). Nucleic acid dye and RNase inhibitor were purchased from Sigma-Aldrich Co, LLC (USA). M-MLV Reverse Transcriptase was supplied by Promega (Beijing) Biotech., Ltd. Marker (100-600 bp) and 2×PCR TaqMix were supplied by Dongsheng Bio (Guangzhou). Finally, diltiazem hydrochloride (DIL) was offered by Shanghai Sine Wanxiang Pharmaceutical Co., Ltd. (China).

\section{Animals}

The experimental procedures and protocols used in this investigation were approved by the Ethics Committee for the Experimental Use of Animals at Guangxi Medical University (Guangxi, China). SD rats $(n=70)$ of both sexes weighing 250-300 g were obtained from the Guangxi Medical University Laboratory Animal Center. The rats were housed under standard conditions $\left(20-25^{\circ} \mathrm{C}, 50-60 \%\right.$ humidity, with a $12 \mathrm{~h}$ light-dark cycle) and were given standard rodent chow and free access to water.

\section{Experimental design}

The seventy Sprague-Dawley rats (rats with normal electrocardiograms selected with an MS4000 biological signal record and analysis system) were randomly divided into the following groups:

I: sham group $(\mathrm{n}=10)$ :

SD rats were subjected to thoracotomy and encircling of the LAD artery with a suture but not ligation 30 minutes after treatment with $2 \mathrm{~mL}$ normal saline/kg body weight;

II: model group ( $\mathrm{n}=10)$ : MI/RI

$\mathrm{SD}$ rats were subjected to ligation of the LAD artery for $30 \mathrm{~min}$, which was then released to allow reperfusion for $1 \mathrm{~h}$;

III: NS group ( $\mathrm{n}=10): \mathrm{MI} / \mathrm{RI}+\mathrm{NS}$

$\mathrm{SD}$ rats were treated with $2 \mathrm{~mL}$ normal saline/kg body weight 30 minutes before subjected to ligation of the LAD artery for $30 \mathrm{~min}$, which was then released to allow reperfusion for $1 \mathrm{~h}$;

IV: DIL group ( $\mathrm{n}=10):$ MI/RI + diltiazem hydrochloride $(5 \mathrm{mg} / \mathrm{kg})$

SD rats were treated with $5 \mathrm{mg}$ diltiazem hydrochloride (diluted with normal saline) / $\mathrm{kg}$ body weight 30 minutes before subjected to ligation of the LAD artery for $30 \mathrm{~min}$, which was then released to allow reperfusion for $1 \mathrm{~h}$;

V: YLSFL group ( $\mathrm{n}=10)$ : MI/RI + YLSF $(20 \mathrm{mg} / \mathrm{kg})$

SD rats were treated with $20 \mathrm{mg}$ YLSF(diluted with normal saline) / kg body weight 30 minutes before subjected to ligation of the LAD artery for $30 \mathrm{~min}$, which was then released to allow reperfusion for $1 \mathrm{~h}$;

VI: YLSFM group ( $\mathrm{n}=10):$ MI/RI + YLSF $(40 \mathrm{mg} / \mathrm{kg})$

SD rats were treated with $40 \mathrm{mg}$ YLSF (diluted with normal saline) / $\mathrm{kg}$ body weight 30 minutes before subjected to ligation of the LAD artery for $30 \mathrm{~min}$, which was then released to allow reperfusion for $1 \mathrm{~h}$;

VII: YLSFH group ( $\mathrm{n}=10): \mathrm{MI} / \mathrm{RI}+\mathrm{YLSF}(80 \mathrm{mg} / \mathrm{kg}$ )

SD rats were treated with $80 \mathrm{mg}$ YLSF (diluted with normal saline) / $\mathrm{kg}$ body weight 30 minutes before subjected to ligation of the LAD artery for $30 \mathrm{~min}$, which was then released to allow reperfusion for $1 \mathrm{~h}$.

The $2 \mathrm{ml} / \mathrm{kg}$ body weight treatments for each group were administered via the duodenum. And diltiazem hydrochloride has an exactly protective effect on MI/RI so DIL group was set up as positive control group.

\section{General surgical procedure}

The MI/RI operation was performed $30 \mathrm{~min}$ after treatment administration. Rats were anesthetized with $20 \%$ ethyl carbamate $(5 \mathrm{ml} / \mathrm{kg}$ body weight $)$ and restrained in the supine position. The chest was opened through the fourth intercostal space, and the ribs were gently retracted to expose the heart. After riving the pericardium, the LAD artery was positioned between the left atrial appendage and the pulmonary conus. The LAD artery was ligated using a 5-0 silk suture. Oxygen was supplied through the trachea using Small Animal Ventilator (Shanghai Alcott Biotech CO., Ltd) with a respiration rate of $70 / \mathrm{min}$, a respirationto-expiration ratio of $1: 2$ and a tidal volume of $50 \mathrm{~mL} / \mathrm{kg}$ body weight. The LAD artery was ligated for 30 
min and released to allow reperfusion for $1 \mathrm{~h}$. The sham group was subjected to an identical treatment omitting the LAD artery ligation. Subcutaneous electrodes were inserted into the limbs of the rats and were monitored by electrocardiogram (ECG) continuously throughout the MI/RI procedure with an MS4000 biological signal record and analysis system. The model was considered successfully established when the ST-segment elevated during myocardial ischemia and dropped at least 50\% during reperfusion [23].

\section{Measurement of myocardial infarction area by Evans blue-TTC staining}

The rats were administered $1.5 \mathrm{ml} 0.5 \mathrm{~g} / \mathrm{L}$ Evans blue dye via thoracic aorta retrograde injection after reperfusion. The heart was removed and washed with pre-cooled saline, and the non-myocardial tissues such as the blood vessels and fat were removed. The left ventricle was sliced into parallel 1- to 1.5-mm-thick myocardial sections. The slices were put into $10 \mathrm{~g} / \mathrm{L} \mathrm{TTC}$ solution (pH 7.4) and incubated at $37^{\circ} \mathrm{C}$ for 15 min. Then, the slices were washed with pre-cooled saline and fixed with $10 \%$ formaldehyde. The infarction area was not stained, the normal area was stained blue, the ischemic area was stained red, and the risk area exhibited a mix of red staining and unstained tissue. Three regions were cut and weighed (weight of infarct, WI; weight of risk, WR; weight of left ventricular, WLV), and the percentages of WI/WLV and WR/WLV were used to reflect the degree of myocardial injury.

\section{Determination of myocardial enzyme levels and lipid peroxidation}

Blood samples were collected from the abdominal aorta after reperfusion, and the serum was immediately separated from the blood samples by centrifugation for $3000 \mathrm{rpm}$ at $4^{\circ} \mathrm{C}$ for $15 \mathrm{~min}$; samples were then stored at $-20^{\circ} \mathrm{C}$. Serum levels of T-SOD, MDA (chromogenic reaction with thiobarbituric acid reactive substances), GSH-Px, AST, CK, CK-MB, LDH and LDH1 were evaluated. The indexes were done according to the manufacturer's instruction.

\section{Assays for NOS activity}

Serum levels of inducible nitric oxide synthase (iNOS), total nitric oxide synthase (tNOS) and constitutive nitric oxide synthase (cNOS) were measured according to the methods described in the manual for the Nitric Oxide Synthase assay kit. It was done according to the manufacturer's instruction.

\section{Analysis of caspase-3 and ANT1 mRNA by RT-PCR}

The heart was promptly removed, washed with cold saline, frozen in liquid nitrogen and preserved at $-80^{\circ} \mathrm{C}$. Total RNA was extracted from ischemic myocardial tissue. The reverse transcription reaction was performed using an RT-PCR kit according to the manufacturer's instructions. The $25 \mu \mathrm{l}$ reaction volume included $2 \mu \mathrm{g}$ of total RNA, and cDNA was synthesized by incubating at $37^{\circ} \mathrm{C}$ for $1 \mathrm{~h}$ and then at $95^{\circ} \mathrm{C}$ for 10 min. The cDNA was stored at $-20^{\circ} \mathrm{C}$. Then, RT-PCR was performed using the cDNA as a template and adding primers, mix and RNase-free water. The amplification product was visualized on a $2 \%$ agarose gel, and the gene bands were analyzed using Quantity One image manipulation software (Versions 4.62, Bio-Rad). The gray value was recorded, and the relative gray value ratio was expressed as the target gene/ $\beta$-actin. The PCR primers are shown below:

ANT1 (171bp, Forward primer: 5'TCTTCAAGTCTGATGGCCTG3', Reverse primer: 5'TCACACTCTGGGCAATCATC3'); caspase-3 (191bp, Forward primer: 5'GAAA GCATCCAGCAATAGGC3', Reverse primer: 5'TGAGTTCCTTCCTTTCTTTGTG C3'); $\beta$-actin (240bp, Forward primer: 5' AACCCTAAGGCCAACCGTGAAAAG 3', Reverse primer: 5' TCATGAGGTAGTCTGTCAGGT 3').

\section{Myocardial histopathology}

The ischemic myocardial samples were fixed in 4\% paraformaldehyde, embedded with paraffin and cut into sections approximately 4-microm thick, followed by routine hematoxylin and eosin (HE) staining. The slides were examined under light microscopy (OLYMPUS, Japan) with a magnification of $400 \times$ by a pathologist who was blind to the experimental profile.

\section{Ischemic myocardium ultrastructure}

Transmission electron microscopy (TEM, Hitachi, Japan) was used to observe the ultrastructure of the myocardium. The myocardia were cut into approximately 70 -nm-thick slices and then fixed in $2.5 \%$ glutaraldehyde and embedded in Epon resin. Then, ultrathin sections were cut and counterstained with uranyl acetate and lead citrate and examined with a Hitachi H-500 TEM. 
Fig. 1. Effect of YLSF on the infarction area and risk area of myocardial tissue. Each column represents the mean $\pm \mathrm{SD}(\mathrm{n}=10)$. ${ }^{*} \mathrm{P}<0.05,{ }^{*} \mathrm{P}<0.01$ compared to the model group, " $\mathrm{P}<0.05$ compared to the sham group, ${ }^{\&} \mathrm{P}<0.05$ compared to the DIL group. Regions stained with different colors were cut and weighed (weight of infarct, WI; weight of risk, WR; weight of left ventricular, WLV). Infarction area is presented as $\mathrm{WI} / \mathrm{WLV}$, and risk area is presented as WR/ WLV. In the sham group, no infarction was observed in the myocardium. The infarc-

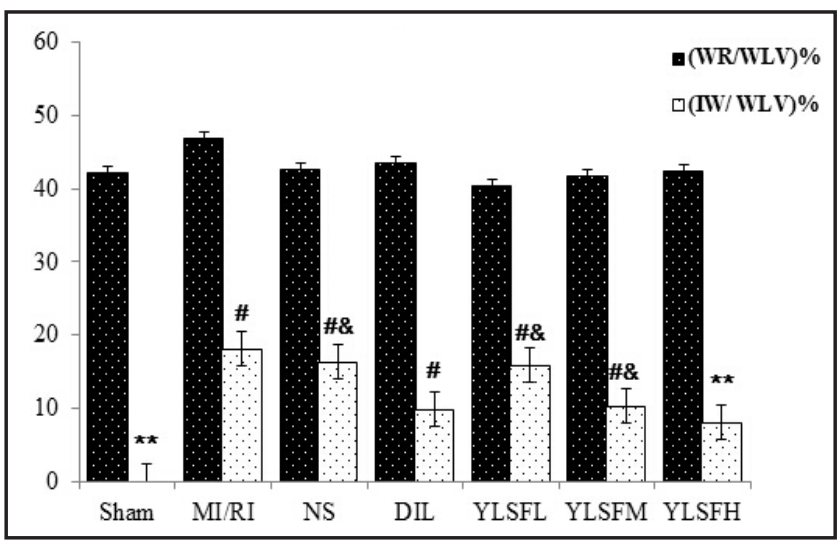
tion area was significantly expanded in the model group and the NS group, and YLSF treatment shranked the infarction area significantly.

Fig. 2. Effect of YLSF on the serum levels of AST, LDH and LDH1 in MI/ RI rats. Each column represents the mean \pm SD $(\mathrm{n}=10) . \quad{ }^{*} \mathrm{P}<0.05$, **P $<0.01$ compared to the model group, " $\mathrm{P}<0.05$ compared to the sham group, ${ }^{\&} P$ $<0.05$ compared to the DIL group.

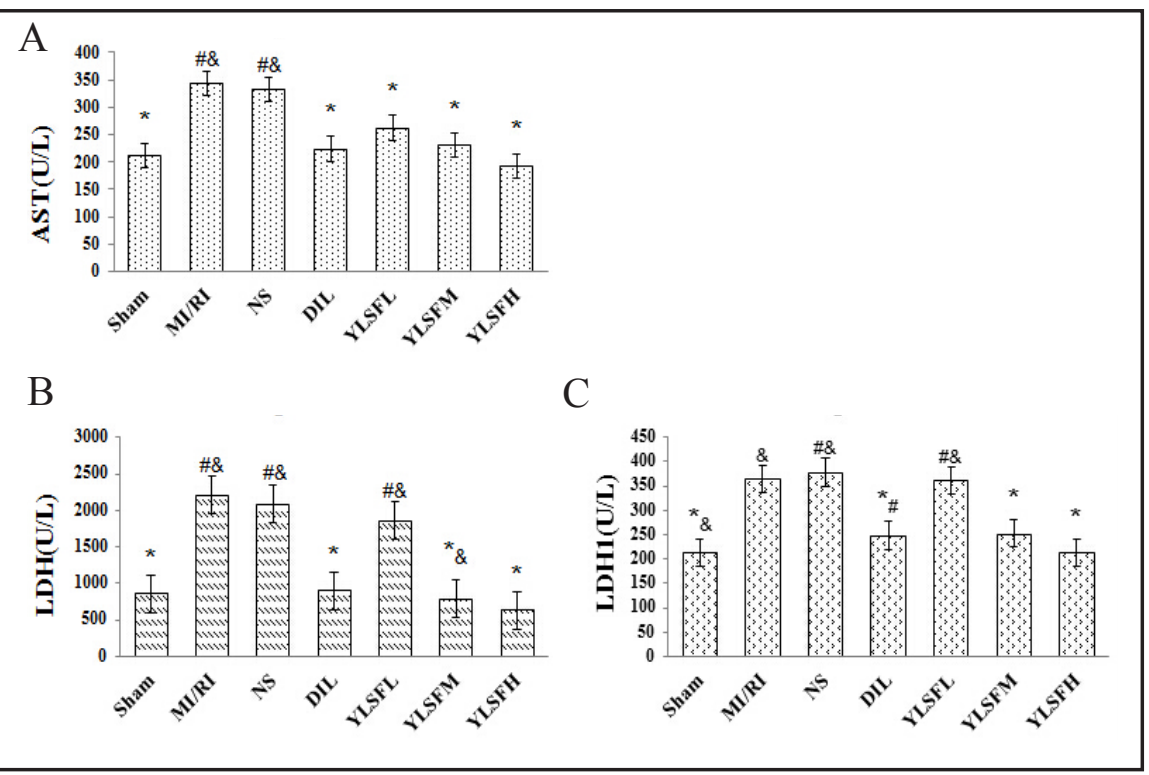

\section{Statistical analysis}

All group values are presented as the means \pm SD, and data were evaluated using the Sigma Stat (version 13.0) statistical analysis program (SPSS Inc, Chicago, IL, USA). Differences between groups were tested for statistical significance using one-way analysis of variance (ANOVA). Differences were considered statistically significant at P-values less than 0.05 .

\section{Results}

YLSF reduced infarct size in rat hearts exposed to ischemia/reperfusion

The infarction area (presented as WI/WLV) was expanded in the model group after MI/RI. As expected, pretreatment with diltiazem, YLSFH $(80 \mathrm{mg} / \mathrm{kg}$ ) or YLSM $(40 \mathrm{mg} /$ $\mathrm{kg}$ ) reduced the infarction area significantly $(\mathrm{P}<0.05$ or $<0.01)$. However, there were no significant differences between the YLSFL group $(20 \mathrm{mg} / \mathrm{kg}$ ) and the model group (YLSFL group: $15.81 \pm 2.84 \%$ vs. model group: $18.17 \pm 4.06 \%, \mathrm{P}>0.05$ ). No myocardial infarction was observed in the sham group. No significant differences were observed in the risk area (presented as WR/WLV) between any of the groups (P>0.05) (Fig. 1). 

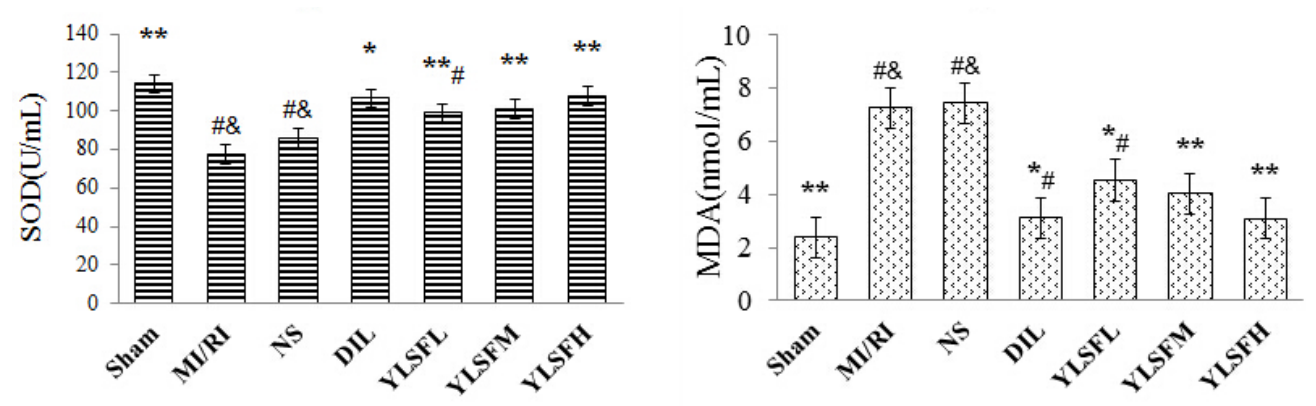

Fig. 3. Effect of YLSF on the serum levels of SOD and MDA. Each column represents the mean \pm SD $(n=10)$. ${ }^{*} \mathrm{P}<0.05$, ${ }^{* *} \mathrm{P}<0.01$ compared to the model group, ${ }^{*} \mathrm{P}<0.05$ compared to the sham group, ${ }^{\&} \mathrm{P}<0.05$ compared to the DIL group.

Fig. 4. Effect of YLSF on the serum levels of GSH-Px, CK and CK-MB. Each column represents the mean \pm SD $(\mathrm{n}=10)$. $* \mathrm{P}<0.05, \quad * * \mathrm{P}$ $<0.01$ compared to the model group, "P $<0.05$ compared to the sham group, ${ }^{\&} \mathrm{P}<0.05$ compared to the DIL group.

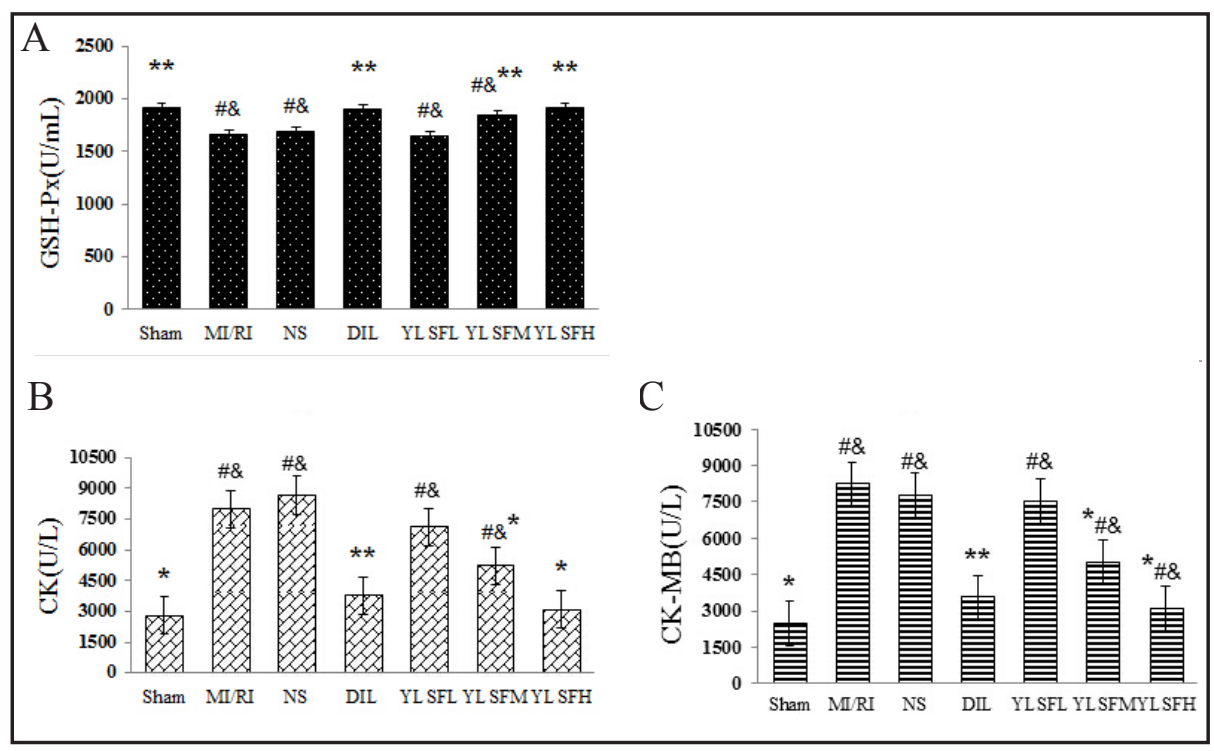

YLSF suppressed the elevation of myocardial enzyme activities and lipid peroxidation in acute MI/RI rats

As a consequence of MI/RI, the serum levels of AST, LDH, LDH1, CK, CK-MB, and MDA were significantly higher in the model group than in the sham group $(\mathrm{P}<0.05$ or $<0.01)$. On the contrary, SOD and GSH-Px activities were significantly lower in the model group than in the sham group $(\mathrm{P}<0.05)$. Compared with the model group, serum levels of AST, LDH, LDH1, $\mathrm{CK}, \mathrm{CK}-\mathrm{MB}$, and MDA were lower in the DIL group and in the three groups pretreated with YLSF $(\mathrm{P}<0.05$ or $<0.01)$. In contrast, SOD and GSH-Px activities were increased remarkably by YLSF treatment compared with the model group, especially in the YLSFH and YLSFM groups $(\mathrm{P}<0.05)$ (Fig. 2-Fig. 4).

Effect of YLSF on serum NOS production during acute MI/RI in rats

Serum tNOS and cNOS activities in the NS group and model group were lower than those in the sham group $(\mathrm{P}<0.05)$, and the serum activity of iNOS in the NS group and model group was higher than that in the sham group $(\mathrm{P}<0.05)$. By pretreating with diltiazem and YLSF $(80 \mathrm{mg} / \mathrm{kg}$ and $40 \mathrm{mg} / \mathrm{kg})$, tNOS and cNOS activities were elevated $(\mathrm{P}<0.05)$ and iNOS activity was reduced $(\mathrm{P}<0.05)$ compared with the model group. However, no significant difference was observed between the YLSF group $(20 \mathrm{mg} / \mathrm{kg})$ and the model group with regard to tNOS, iNOS and cNOS activity $(\mathrm{P}>0.05)$ (Fig. 5). 
Fig. 5. Effect of YLSF on tNOS, iNOS and cNOS activities. Each column represents the mean \pm SD $(\mathrm{n}=10) .{ }^{*} \mathrm{P}<0.05$, $* * \mathrm{P}<0.01$ compared to the model group, \#P $<0.05$ compared to the sham group, \& $\mathrm{P}<0.05$ compared to the DIL group.
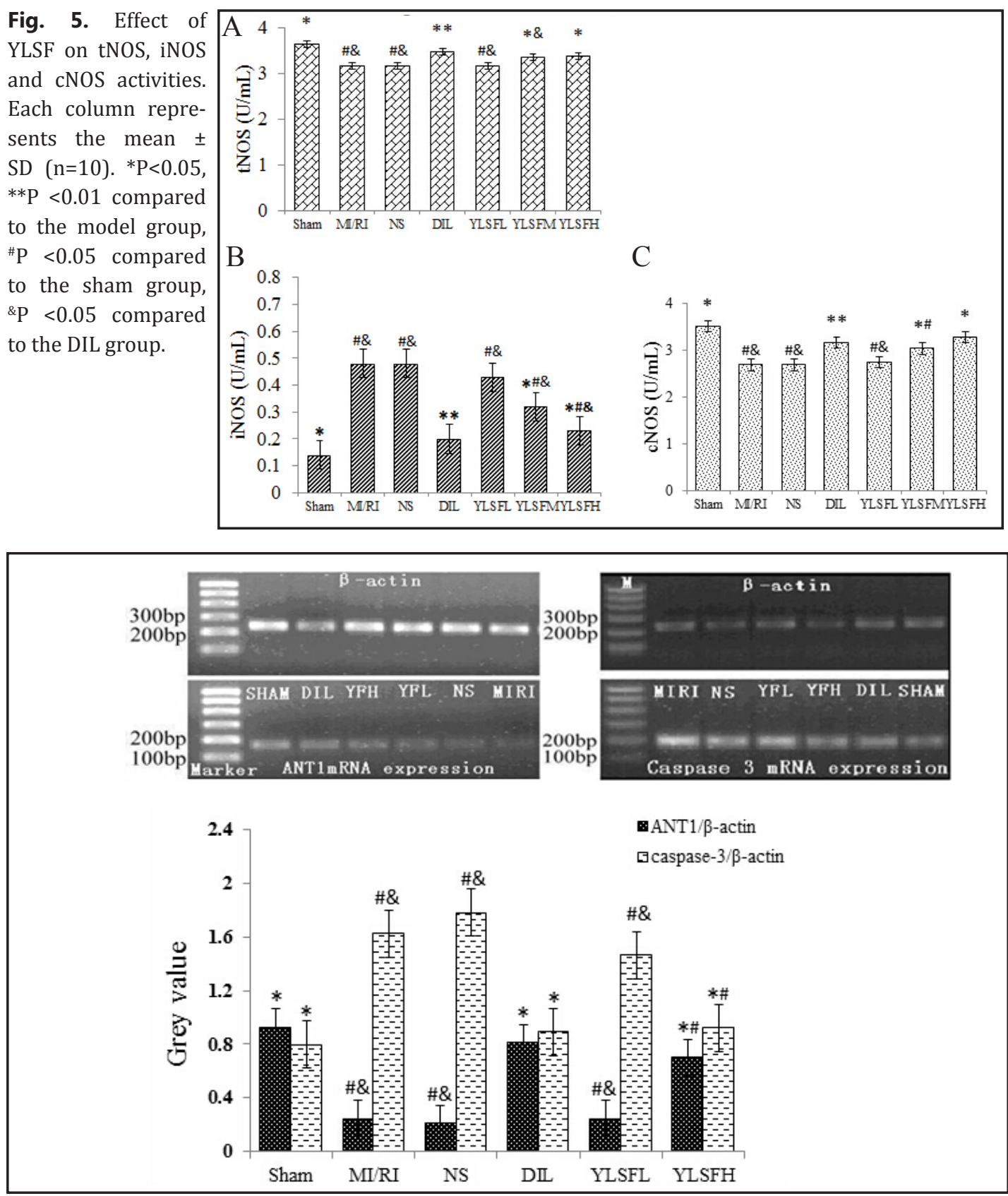

Fig. 6. Effect of YLSF on ANT1 and caspase- 3 mRNA expression. Each column represents the mean \pm SD $(\mathrm{n}=8) .{ }^{*} \mathrm{P}<0.05,{ }^{* *} \mathrm{P}<0.01$ compared to the model group, ${ }^{*} \mathrm{P}<0.05$ compared to the sham group, ${ }^{\&} \mathrm{P}<0.05$ compared to the DIL group.

Effect of YLSF on the expression of apoptosis-related genes

Caspase-3 and ANT1 mRNA expression levels were measured using Quantity One software using the grey value expressed as the ratio of caspase- $3 / \beta$-actin and ANT $1 / \beta$-actin, respectively. As shown in Fig. 6, amplification bands of ANT1 mRNA and caspase-3 mRNA can be observed at $171 \mathrm{bp}$ and $191 \mathrm{bp}$, respectively. Pretreatment with diltiazem or YLSFH $(80 \mathrm{mg} / \mathrm{kg}$ ) both increased the expression of ANT1 mRNA compared with the model group $(\mathrm{P}<0.05)$, and there was no significant difference between the YLSF groups ( 40 and $20 \mathrm{mg} /$ $\mathrm{kg}$ ) and model group ( $\mathrm{P}>0.05)$. In YLSFH group $(80 \mathrm{mg} / \mathrm{kg})$ and DIL group the expression of 
Fig. 7. Effect of YLSF pretreatment on histopathological alterations $(\times 200)$.

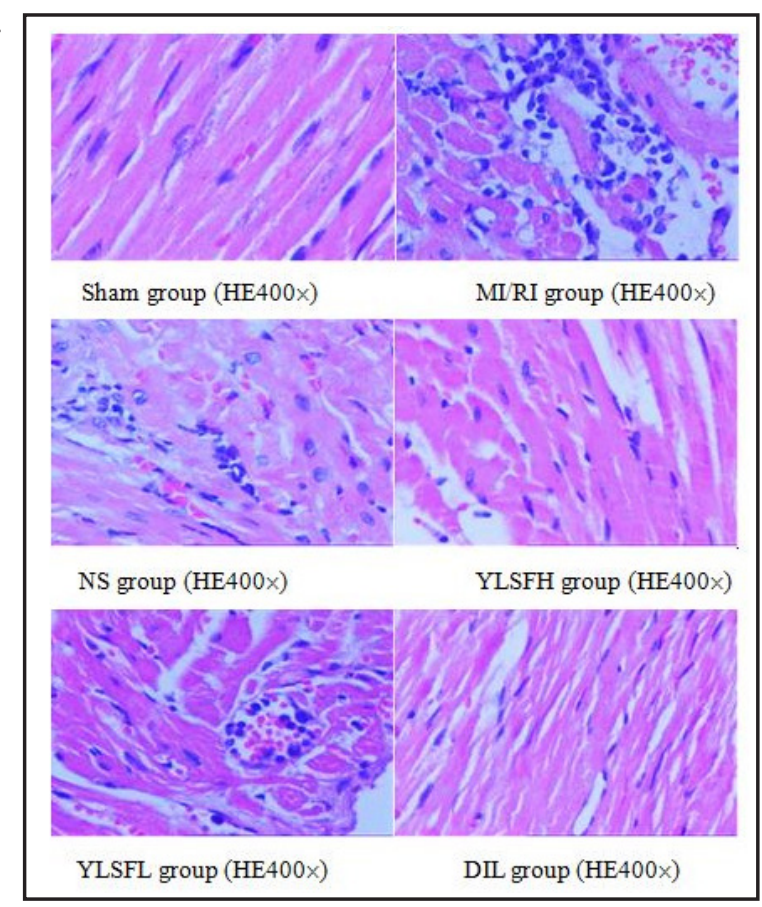

caspase-3 mRNA was decreased compared with the model group $(\mathrm{P}<0.05)$, and there was no significant difference between the YLSF groups $(40$ and $20 \mathrm{mg} / \mathrm{kg}$ ) and the model group $(\mathrm{P}>0.05)$ (Fig. 6).

\section{Effect of YLSF pretreatment on histopathological alterations}

As shown in Fig. 7, histopathological examination of the myocardium of a rat from the sham group showed a uniform color and an intact myocardial histology without evidence of myocardial membrane damage. The model group showed uneven staining, with an enlarged interstitial region, a small amount of inflammatory cell infiltration around the interstitial region and a small hemorrhage. Treatment with YLSF generally resulted in improved pathological results compared to the model group and showed a trend of decreasing myonecrosis, infiltration of inflammatory cells, extravasation of red blood cells and edema. The DIL group showed similar results as the sham group except the DIL group had a higher degree of edema in the myocardial intercellular space.

\section{Effect of YLSF pretreatment on ultrastructural alterations}

The myocardia of the model group exhibited serious degeneration and necrosis in which the cell membranes were destroyed, the mitochondria were overly varicose and decreased, the myofilaments were soluble, and contractive band necrosis appeared. The myocardia of the model group were arranged regularly, with clear and integrated mitochondrial structures, intercalated discs and myofilaments. Compared with the model group, pathologic changes in the YLSF groups were less severe. The muscle fibers were not as irregularly arranged and exhibited less dissolution. There was also less swelling and disruption of the mitochondria and fewer abnormal mitochondria in terms of shape and size. The degree of pathological injury was less severe in the DIL group than in the YLSFH group, but it still showed some mitochondrial swelling and cell membrane effusion (Fig. 8).

\section{Discussion}

Ligation of the left anterior descending coronary artery is a classical method for establishing the MI/RI model in animals. In our present research, the risk area (WR/ 


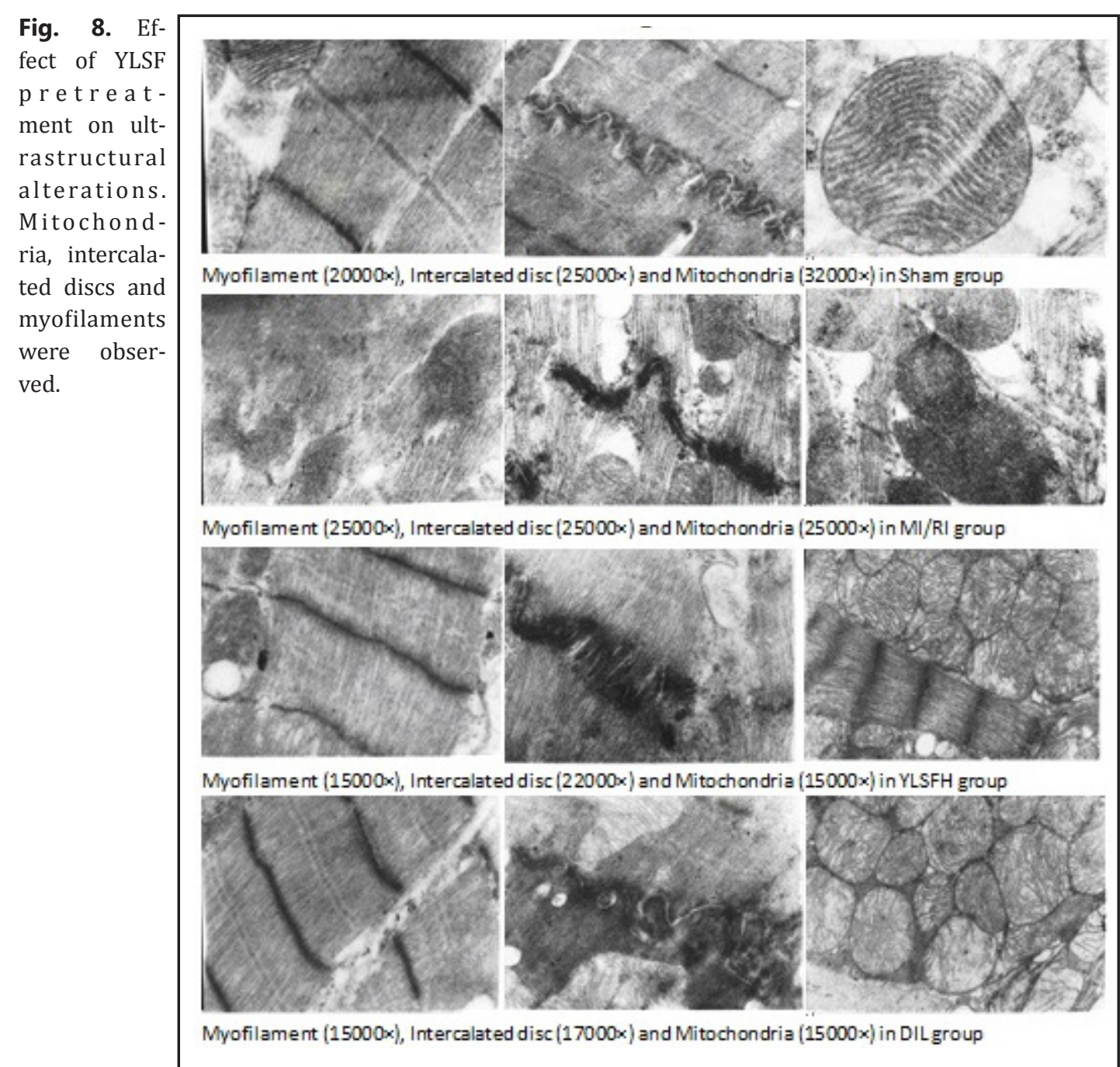

WLV) in each group showed no significant difference $(P>0.05)$ compared with the model group, which could imply that the ligation portion of the procedure was basically consistent across the study groups. The infarction area (WI/ WLV) in the sham group and the YLSFH and YLSFM groups was decreased dramatically compared with the model group, and no significant difference was found between the model group and NS group, with the addition of ST-segment elevating during myocardial ischemia and dropping at least $50 \%$ during reperfusion, which could imply that a successful rat MI/RI model was established [23]. In summary, we concluded that pretreatment with YLSF could decrease the infarction area of $\mathrm{MI} / \mathrm{RI}$ rats and protect against injury to the myocardium.

Plentiful evidence demonstrates that the pathogenesis of MI/RI can be divided into several phases [24]. The initial injury is triggered by reactive oxygen species (ROS) with inflammation involving chemokines and cytokines, followed by neutrophil-mediated myocardial injury occurring in the late period of reperfusion [25]. A large number of reported studies suggest that ROS are a main contributing factor of MI/RI, and ROS may injure cells by causing membrane lipid peroxidation, consequently leading to apoptosis or death in the process of MI/RI [26, 27]. Therefore, further investigation was performed with a focus on the antioxidant properties involved in the cardioprotective effects of YLSF. As an endogenous antioxidant enzyme, SOD plays an important role in protecting the cells from oxidative damage by converting $\mathrm{O}^{2-}$ into $\mathrm{H}_{2} \mathrm{O}_{2}$, and $\mathrm{GSH}$ is an important non-enzymatic antioxidant defense process required to maintain the normal redox state of cells and to counteract the deleterious effects of oxidative stress [28]. Our results showed that the significant decrease 
in the GSH-Px and SOD levels in the model group was enhanced by YLSF, which indicated the potential of YLSF to counteract oxidative damage and to reinforce antioxidant defenses. Moreover, when the cell membrane becomes permeable, a variety of intracellular enzymes are released into the blood during MI/RI, such as CK, CK-MB, LDH and LDH1. These enzymes are considered diagnostic markers of myocardial tissue damage [29-31]. Our research showed a significant elevation in the levels of CK, CK-MB, LDH and LDH1 in the model group and demonstrated that pretreatment with YLSF decreased the levels of these enzymes. The mechanism for this reduction might be related to maintenance of the membrane integrity of myocardial cells.

Some animal studies have indicated that myocardial infarction enhances the activity of iNOS and that the abnormal increase of .NO participates in the process of myocardial injury. These studies have presented a positive correlation between iNOS activity and the myocardial infarction area [32]. In addition, increased iNOS activity aggravates myocardial ischemia/reperfusion injury. The function of the infarcted left ventricle is ameliorated and the infarction area is reduced by blocking iNOS. In addition, the infarcted left ventricular systolic function is improved and apoptosis is decreasing by iNOS gene knock out [33-35]. Wang also found that the increased iNOS activity is one of the significant mechanisms during MI/RI. However, there were two contradictory viewpoints on the protective effect and toxic effect about iNOS [36]. Many researches demonstrated that drugs against myocardial infarction by overexpression of iNOS $[37,38]$. On the contrary, increased cNOS activity is capable of antagonizing MI/RI [39]. Our study also demonstrates that iNOS activity is increased during $\mathrm{MI} / \mathrm{RI}$ and cNOS activity is increased by pretreatment with YLSF.

ANT is the most abundant protein in the myocardial mitochondrial membrane with species differences, and its isomerase exists in different tissues and is encoded by a corresponding nuclear gene [40]. There are two ANT isomerases in rats and mice, namely ANT1 isomerase and ANT2 isomerase, with strict tissue specificity. ANT1 is dominant in the myocardium [41]. ANT1 is decreased during MI/RI, and reactive oxygen species levels distinctly increased when the myocardium lacks ANT1 [42, 43]. In our study, we found that pretreatment with YLSFH $(80 \mathrm{mg} / \mathrm{kg}$ ) increased the expression of ANT1 mRNA compared with the model group $(\mathrm{P}<0.05)$, which was in accordance with the findings above. Caspase is a proteinase that is relevant to the maturity of cytokines and cell apoptosis. In addition, caspase- 3 is the key enzyme of apoptosis in mammals, and various stimulating factors can lead to the activation of caspase-3, including MI/RI [44]. The proteins of the cytoplasm, karyon and cytoskeleton are degraded and deactivated. The present study shows that YLSF decreased the expression of caspase-3, whereas caspase-3 expression in the model group dramatically increased, implying that the inhibition of apoptosis may be one of the mechanisms by which YLSF protects against MI/RI.

In our present study, we found that YLSF can protect the heart from MI/RI as evidenced by the improved ultrastructure of the mitochondria, myofilaments and intercalated discs. In addition, the histopathological examination demonstrated apparent myocardial damage in the model group compared with the sham group. Extensive cloudy swelling, hyperemia, sinusoidal distension and focal vacuolar degeneration were also observed in the model group. Our results indicated that YLSF restored most of these pathological heart lesions, thereby demonstrating the compound's cardioprotective action. Infarction area is considered a key criterion for estimating MI/RI injury and is directly related to the prognosis of patients in clinic ${ }^{[45]}$. It is worth noting that YLSF can significantly reduce the myocardial infarct area compared with the model group. There was no significant difference in the risk area (presented as WR/WLV) between YLSF group and the model group $(\mathrm{P}>0.05)$.

In conclusion, our study showed that YLSF increases T-SOD, GSH-Px, ANT1 and cNOS levels and decreases the infarction area and caspase-3, iNOS, MDA, AST, CK, CK-MB, LDH and LDH1 levels. In addition, YLSF improves the histopathological characteristics and the ultrastructure of the tissue. These results imply that reducing lipid peroxidation damage, regulating the activity of NOS and modulating the expression of genes associated with apoptosis by pretreating with YLSF is a feasible therapeutic strategy for treating MI/RI. 
Therefore, YLSF can be considered a promising therapeutic agent for the prevention and treatment of MI/RI.

\section{Abbreviations}

ANT1 (adenine nucleotide translocator-1); AST(aspartate aminotransferase); cNOS (constitutive nitric oxide synthase); CK (creatine kinase); CK-MB (creatine kinase-MB); DIL (diltiazem hydrochloride); GSH-Px (glutathione peroxidase); iNOS (inducible nitric oxide synthase);LDA (left anterior descending); LDH (lactate dehydrogenase); LDH1 (l a c t a t e dehydrogenase1); MI/RI (myocardial ischemia reperfusion injury); MDA (malondialdehyde); .NO (nitric oxide); NOS (nitric oxide synthase); ROS (reactive oxygen species); RT-PCR (reverse transcription polymerase chain reaction); SOD (superoxide dismutase); tNOS(total nitric oxide synthase); TTC (tetrazolium chloride); WI (weight of infarct); WLV (weight of left ventricular); WR (weight of risk); YLSF (Yulangsan Flavonoid).

\section{Disclosure Statement}

The authors declare that there are no conflicts of interest.

\section{Acknowledgements}

This work was supported by the Guangxi Scientific Research and Technology Development Research Projects (No. 0630002-2A); the Guangxi Natural Science Foundation (No. 2013GXNSFAA019175); the Fund of Guangxi Key Laboratory of Functional Phytochemicals Research and Utilization (No. FPRU2013-3); and the Outstanding Doctoral Dissertation breeding program in Guangxi Zhuang Autonomous Region (No. YCBZ2012013).

\section{References}

- Sekikawa A, Horiuchi BY, Edmundowicz D, Ueshima H, Curb JD, Sutton-Tyrrell K, Okamura T, Kadowaki T, Kashiwagi A, Mitsunami K, Murata K, Nakamura Y, Rodriguez BL, Kuller LH: A "natural experiment" in cardiovascular epidemiology in the early 21st century. Heart 2003;89:255-257.

2 Jennings RB, Sommers HM, Smyth GA, Flack HA,LinnI H: Myocardial necrosis induced by temporary occlusion of a coronary artery in the dog. Arch Pathol 1960;70:68-78.

-3 Lee YM, Cheng PY, Chen SY, Chung MT, Sheu JR: Wogonin suppresses arrhythmias, inflammatory responses, and apoptosis induced by myocardial ischemia/reperfusion in rats. J Cardiovasc Pharm 2011;58:133-142.

-4 Soloviev A, Stefanov A, Parshikov A, Khromov A, Moibenko A, Kvotchina L, Balavoine G, Geletii Y: Arrhythmogenic peroxynitrite-induced alterations in mammalian heart contractility and its prevention with quercetin-filled liposomes. Cardiovasc Toxicol 2002;2:129-139.

5 Bishopric NH, Andreka P, Slepak T, Webster KA: Molecular mechanisms of apoptosis in the cardiac myocyte. Curr Opin Pharmacol 2001;1:141-150.

6 Gustafsson AB, Gottlieb RA: Mechanisms of apoptosis in the heart. J Clin Immunol 2003;23:447-459.

7 Bruegger D, Rehm M, Jacob M, Chappel D, Stoeckelhuber M, Welsch U, Conzen P, Becker BF: Exogenous nitric oxide requires an endothelial glycocalyx to prevent postischemic coronary vascular leak in guinea pig hearts. Crit Care 2008;12:73. 
8 Hirai DM, Copp SW, Ferreira LF, Musch TI, Poole DC: Nitric oxide bioavailability modulates the dynamics of microvascular oxygen exchange during recovery from contractions. ACTA Physiol 2010;200:159-169.

-9 Moncada S, Palmer RM, Higgs EA: Nitric oxide: physiology, pathophysiology, and pharmacology. Pharmacol Rev 1991;43:109-142.

10 Förstermann U, Closs EI, Pollock JS, Nakane M, Schwarz P, Gath I, Kleinert H: Nitric oxide synthase isozymes. Characterization, purification, molecular cloning, and functions. Hypertension 1994;23:11211131.

11 McGowan FX Jr, Davis PJ, del Nido PJ, Sobek M, Allen JW, Downing SE: Endothelium-dependent regulation of coronary tone in the neonatal pig. Anesth Analg 1994;79:1094-1101.

12 Radomski MW, Palmer RM, Moncada S: The anti-aggregating properties of vascular endothelium: interactions between prostacyclin and nitric oxide. Brit J Pharmacol 1987;92:639-646.

13 Kubes P, Suzuki M, Granger DN: Nitric oxide: an endogenous modulator of leukocyte adhesion. P Natl Acad Sci USA 1991;88:4651-4655.

14 Groves PH, Lewis MJ, Cheadle HA, Penny WJ: SIN-1 reduces platelet adhesion and platelet thrombus formation in a porcine model of balloon angioplasty. Circulation 1993;87:590-597.

15 Radomski MW, Palmer RM, Moncada S: Endogenous nitric oxide inhibits human platelet adhesion to vascular endothelium. Lancet 1987;2:1057-1058.

16 Kim YM, Bombeck CA, Billiar TR: Nitric oxide as a bifunctional regulator of apoptosis. Circ Res 1999;84:253-256.

17 Brunner F, Maier R, Andrew P, Wölkart G, Zechner R, Mayer B: Attenuation of myocardial ischemia/ reperfusion injury in mice with myocyte-specific overexpression of endothelial nitric oxide synthase. Cardiovasc Res 2003;57:55-62.

18 Department of Health of Guangxi Zhuang Autonomous Region: Guangxi Chinese medicine standard. Guangxi Science and Technology Press. 1992, vol 35.

19 Huang XC, Zhou ZC, Zhang J: Guangxi national medicine compendium. Guangxi Institute for Drug Control 1980, vol 134.

20 Zhang XD, Jiang WZ, Jiao Y, Huang RB: The Study of Longyanshen Flavonoids on Anti- lipid Peroxidation Injury Induced by Myocardial Ischemia Reperfusion in Rats. Lishizhen Med Mater Med Res 2008;19:607609.

21 Huang RB, Lv JH, Zhang XD, Fv SJ, Jiao Y: Effects of Yulangsan flavonoids on myocardial ischemia reperfusion injury in isolated rat hearts. West China J Pharm Sci 2010;25:287-289.

22 Jian J, Huang JC, Jiao Y, Tan HD, Huang RB: Isolation and preparation of chalcone compounds from tuber of Millettia pulchra var. laxior by pre-HPLC. Chin Tradit Herb Drugs 2011;42:1313-1316.

23 Jy W, Yan N, Yang D-h: Electrocardiogram changes on myocardial ischenmia-reperfusion model in rats. South China J Cardiovasc Dis 2011;17:503-506.

24 Yellon DM, Hausenloy DJ: Myocardial reperfusion injury. N Engl J Med 2007;357:1121-1135.

25 Dhalla NS, Elmoselhi AB, Hata T, Makino N: Status of myocardial antioxidants in ischemia-reperfusion injury. Cardiovasc Res 2000;47:446-456.

-26 Peterson DA, Asinger RW, Elsperger KJ, Homans DC, Eaton JW: Reactive oxygen species may cause myocardial reperfusion injury. Biochem Biophys Res Commun 1985;127:87-93.

-27 Venardos KM, Perkins A, Headrick J, Kaye DM: Myocardial ischemia-reperfusion injury, antioxidant enzyme systems, and selenium: a review. Curr Med Chem 2007;14:1539-1549.

28 Kupatt C, Hinkel R, Horstkotte J, Deiss M, von Brühl ML, Bilzer M, Boekstegers P: Selective retroinfusion of GSH and cariporide attenuates myocardial ischemia-reperfusion injury in a preclinical pig model. Cardiovasc Res 2004;61:530-537.

29 Gürgün C, Ildizli M, Yavuzgil O, Sin A, Apaydin A, Cinar C, Kültürsay H: The effects of short term statin treatment on left ventricular function and inflammatory markers in patients with chronic heart failure. Int J Cardiol 2008;123:102-107.

- 30 Dörner A, Schulze K, Rauch U, Schultheiss HP: Adenine nucleotide translocator in dilated cardiomyopathy: pathophysiological alterations in expression and function. Mol Cell Biochem 1997;174:261-269.

-31 Kirshenbaum LA, Singal PK: Changes in antioxidant enzymes in isolated cardiac myocytes subjected to hypoxia-reoxygenation. Lab Invest 1992;67:796-803. 
-32 Wang D, Yang XP, Liu YH, Carretero OA, Lapointe MC: Reduction of myocardial infarct size by inhibition of inducible nitric oxide synthase. Am J Hypertens 1999;12:174-182.

-33 Saito T, Hu F, Tayara L, Fahas L, Shennib H, Giaid A: Inhibition of NOS II prevents cardiac dysfunction in myocardial infarction and congestive heart failure. Am J Physiol Heart Circ Physiol 2002;283:339-345.

-34 Wildhirt SM1, Weismueller S, Schulze C, Conrad N, Kornberg A, Reichart B: Inducible nitric oxide synthase activation after ischemia/reperfusion contributes to myocardial dysfunction and extent of infarct size in rabbits: evidence for a late phase of nitric oxide-mediated reperfusion injury. Cardiovasc Res 1999;43:698711.

-35 Feng Q Lu X, Jones D L, Shen J, Arnold JM: Increased inducible nitric oxide synthase expression contributes to myocardial dysfunction and higher mortality after myocardial infarction in mice. Circulation 2001;104:700-704.

36 Mehlhorn U, Bloch W, Krahwinkel A, LaRose K, Geissler HJ, Hekmat K, Addicks K, de Vivie ER: Activation of myocardial constitutive nitric oxide synthase during coronary artery surgery. Eur J Cardiothorac Surg 2000;17:305-11.

- 37 Zaitone SA, Abo-Gresha NM: Rosuvastatin promotes angiogenesis and reverses isoproterenol-induced acute myocardial infarction in rats: role of iNOS and VEGF. Eur J Pharmacol 2012;691:134-142.

38 Ma LL, Zhang FJ, Yan M: Sevoflurane preconditioning produces delayed cardioprotection effect through upregulation of inducible nitric oxide synthase in rats. J Zhejiang Univ 2012;41:553-558.

-39 Wang W, Sawichi G, Schulz R: Peroxynitrite-induced myocardial injury is mediated through matrix metalloproteinase-2. Cardiovasc Res 2002;53:165-174.

-40 Doerner A, Pauschinger M, Badorff A, Noutsias M, Giessen S, Schulze K, Bilger J, Rauch U, Schultheiss HP: Tissue-specific transcription pattern of the adenine nucleotide translocase isoforms in humans. FEBS Lett 1997;414:258-262.

-41 Dorner A, Olesch M, Giessen S, Pauschinger M, Schultheiss HP: Transcription of the adenine nucleotide translocase isoforms in various types of tissues in the rat. Biochim Biophys Acta 1999;1417:16-24.

-42 Ning XH, Xu CS, Song YC, Xiao Y, Hu YJ, Lupinetti FM, Portman MA: Hypothermia preserves function and signaling for mitochondrial biogenesis during subsequent ischemia. Am J Physiol 1998;274:786-793.

43 Esposito LA, Melov S, Panov A, Cottrell BA, Wallace DC: Mitochondrial disease in mouse results in increased oxidative stress. Proc Natl Acad Sci USA 1999;96:4820-4825.

-44 Lakhani SA, Masud A, Kuida K, Porter GA, Booth CJ, Mehal WZ, Inayat I, Flavell RA: Caspases 3 and 7: key mediators of mitochondrial events of apoptosis. Science 2006;311:847-851.

-45 Hataishi R, Rodrigues AC, Neilan TG, Morgan JG, Buys E, Sshiva S, Tambouret R, Jassal DS, Raher MJ, Furutani E, Ichinose F, Gladwin MT, Rosenzweig A, Zapol WM, Picard MH, Bloch KD, Scherrer-Crosbie M: Inhaled nitric oxide decreases infarction size and improves left ventricular function in a murine model of myocardial ischemia-reperfusion injury. Am J Physiol Heart Circ Physiol 2006;291:379-384. 